\title{
Sharp Electroluminescence Lines Excited by Tunneling Injection Into a Large Ensemble of Quantum Dots
}

\author{
A. Baumgartner, L. Turyanska, A. Chaggar, A. Patanè, L. Eaves and M. Henini \\ School of Physics and Astronomy, University of Nottingham, Nottingham NG7 2RD, UK
}

\begin{abstract}
We observe a strong bias-dependence of the electroluminescence spectra of an ensemble of self-assembled InAs quantum dots (QDs) excited by tunnelling injection of carriers from the $n$ - and $p$-doped GaAs layers of a $p$ - $i-n$ diode. We show that the dot emission evolves from a broad band above flat-band condition to a series of sharp emission lines below a characteristic bias voltage. Also, we present a study of the electroluminescence under resonant bias excitation of the dots and demonstrate up-conversion luminescence.
\end{abstract}

Keywords: Electroluminescence excitation, self-assembled InAs quantum dots, tunnelling injection PACS: 73.21.La, 73.40.Kp, 73.43.Jn

\section{INTRODUCTION}

Self-assembled InAs quantum dots (QDs) are artificial nanostructures with discrete and narrow electronic energy levels [1]. High-resolution spatially-resolved optical techniques, such as near-field scanning optical microscopy and micro-photoluminescence, have been extensively used to study individual dots. Selective emission from a small number of dots within a large ensemble has also been achieved by incorporating the dots in lithographically patterned small area diodes [14], microcavities pillars [5] and nanowires [6]. Such studies have provided information about the electronic properties of the dots and are crucial for novel applications in spintronics [7] and quantum information processing [8]. In this work, we report sharp electroluminescence (EL) lines and upconversion luminescence (UCL) from individual QDs excited by resonant tunnelling injection of carriers from the $n$ - and $p$-doped GaAs layers of a $p$ - $i$ - $n$ diode.

\section{EXPERIMENTAL DETAILS}

Our $p$-i-n diode was grown by Molecular Beam Epitaxy and incorporates a single layer of selfassembled InAs QDs in the intrinsic (i) GaAs region. The spacer layers between the dots and the contact layers are $100 \mathrm{~nm}$ and $60 \mathrm{~nm}$ wide on the $n$ - and $p$-side of the diode, respectively. The density of QDs is $\sim 10^{11} \mathrm{~cm}^{-2}$. The QDs have mean diameter of $\sim 20 \mathrm{~nm}$ and mean height of $\sim 2 \mathrm{~nm}$ [9]. The $p-i-n$ structure was processed into a circular mesa of $400 \mu \mathrm{m}$ diameter and contains an ensemble of $\sim 10^{8}$ QDs. A ring-shaped electrical contact was fabricated on top of the mesa to permit optical access to the sample for measurements of EL and current-voltage (I-V) characteristics at $4.2 \mathrm{~K}$.

\section{RESULTS}

Figure 1(a) shows a series of EL spectra at various applied voltages, $V$. At $V=1.56 \mathrm{~V}$, well above the flat band condition at $\sim 1.50 \mathrm{~V}$, the QD EL spectrum has approximately Gaussian shape. With decreasing $V$, the EL intensity decreases and the QD EL band evolves into a series of sharp lines as $V$ is decreased below a characteristic voltage $V^{*} \approx 1.40 \mathrm{~V}$. At the lowest voltages $(V \sim 1.2 \mathrm{~V})$, we observe sharp EL lines with line widths limited by the resolution of our spectrometer. At these low voltages, the applied bias provides a means of tuning the electron and hole injection into a small number of QDs within the large ensemble [9]. As shown schematically in Fig. 2, the electrons and holes can enter the QDs either directly by tunnelling from the $p$ - and $n$ - doped contacts (through the potential barriers due to the intrinsic GaAs layers) into the respective ground states, or else via the states of the InAs wetting layer (WL) subband. This also leads to a well defined resonant feature in the the normalised differential conductance $(\mathrm{d} I / \mathrm{d} V) I^{1}$ at $V \approx 1.2 \mathrm{~V}$ (see Figure 1(b)). These bias conditions correspond to an enhancement of the current flow due to tunnelling of electrons and holes into the dots. 
(a)

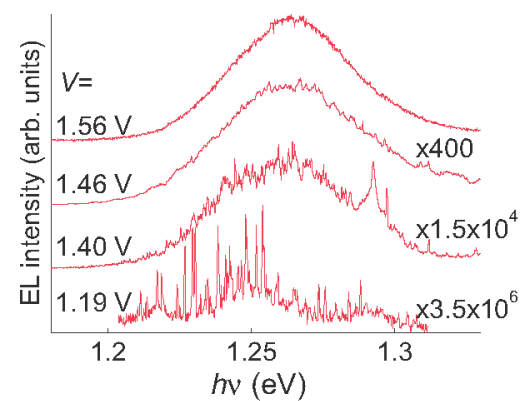

(b)

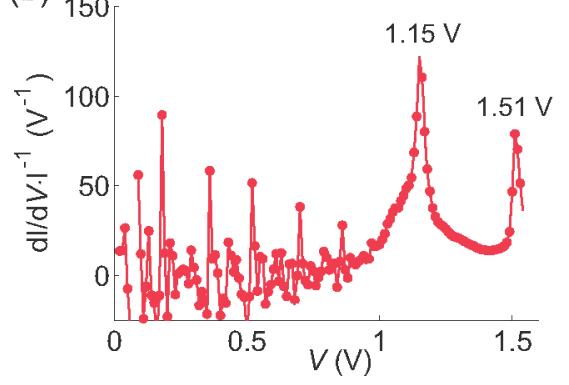

Figure 1. (a) Normalised EL spectra for a series of bias voltages, $V$. (b) Normalised differential conductance $(\mathrm{d} / / \mathrm{d} V) I^{1}$ as a function of $V(T=4.2 \mathrm{~K})$.

We now consider the series of EL lines corresponding to resonant excitation of the QD ground states, see Figure 1(a). We have observed up-conversion luminescence (UCL), i.e. emission from the dots at energies higher than the excitation energy provided by the applied bias. Up-conversion luminescence in InAs self-assembled QDs has already been observed under resonant excitation of the dots by photons [10-11]. However, to our knowledge it was never observed in a $p-i-n$ diode incorporating QDs. In previous works [1011], the UCL was tentatively attributed to a twophoton absorption process. In our samples, electrons and holes are injected electrically into the dots and other mechanisms should therefore be responsible for the UCL. Auger-like mechanisms involving the ground and excited states of the QDs could explain this unusual UCL (Fig. 2d) and require further investigation.

\section{CONCLUSIONS}

We reported sharp electroluminescence lines from a small number of InAs self-assembled QDs excited by resonant tunnelling injection of carriers from the $n$ and $p$-doped layers of $p-i-n$ diodes. Bias-tunable tunnelling of carriers provides us with a means of controlling injection and light emission without resorting to small area lithography. We also observed up-conversion electroluminescence from the dots. (a)
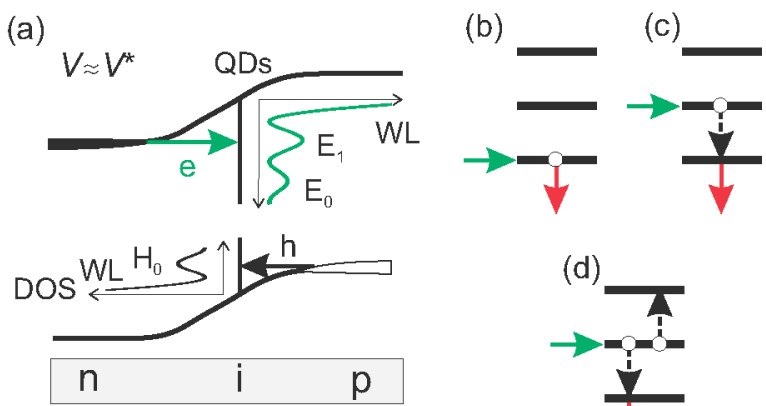

(d)

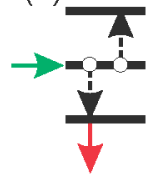

Figure 2. (a) Schematic of electron and hole injection into the dot from the $n$ and $p$-side of the diode. (b) Resonant electron injection into the ground state of a QD followed by electron-hole recombination; (c) injection of an electron into an excited QD state followed by relaxation to the ground state; (d) Auger-like process and up-conversion luminescence.

\section{ACKNOWLEDGMENTS}

This work is supported by the EPSRC (UK) and the European SANDiE Network of Excellence.

\section{REFERENCES}

[1] D.J. Mowbray and M.S. Skolnick, J. Phys. D- App1. Phys. 38, 2059 (2005).

[2] I. E. Itskevich, S. I. Rybchenko, I. I. Tartakovskii, S.T. Stoddart, A. Levin, P. C. Main, L. Eaves, M. Henini, and S. Parnell, Appl. Phys. Lett. 76, 3932 ( 2000).

[3] Z. Yuan, B.E. Kardynal, R.M. Stevenson, A.J. Shields, C. J. Lobo, K. Cooper, N. S. Beattie, D. A. Ritchie, and M. Pepper, Science 295, 102 (2002).

[4] X.L. Xu, D.A. Williams, and J.R.A. Cleaver, Appl. Phys. Lett. 85, 3238 (2004).

[5] P. Michler, A. Kiraz, C. Becher, W.V. Schoenfeld, P.M. Petroff, L.D. Zhang, E. Hu, and A. Imamoglu, Science 290, $2282(2000)$.

[6] M.T. Borgstrom, V. Zwiller, E. Muller, and A. Imamoglu, Nano Lett. 5, 1439 (2005).

[7] M. Kroutvar, Y. Ducommun, D. Heiss, M. Bichler, D. Schuh, G. $\Lambda$ bstreiter, and J.J. Finley, Nature 432, 81 (2004).

[8] S. Stufler, P. Ester, A. Zrenner, and M. Bichler, Phys. Rev. Lett. 96, 037402 (2006).

[9] L. Turyanska, A. Baumgartner, A. Chaggar, A. Patanè, L. Eaves and M. Henini, submitted to Appl. Phys. Lett.

[10] P.P. Paskov, P.O. Holtz, B. Monemar, J.M. Garcia, W.W. Schoenfeld, P.M. Petroff, Appl. Phys. Lett. 77, $812(2000)$.

[11] C. Kammerer, G. Cassabois, C. Voisin, C. Delalande, P. Roussignol, and J.M. Gerard, Phys. Rev. Lett. 87, 207401 (2001). 